\title{
A Survey on Multicast Routing Protocols for Mobile Ad Hoc Networks
}

\author{
Shaveta Jain \\ Department of Computer Science \& Engineering, \\ Maharishi Markandeshwar University, \\ Mullana, Ambala
}

\begin{abstract}
There are many benefits of multicasting using with network. The communication cost reduced by multicasting for applications that sends the same data to many recipients instead of sending via multiple unicast. This paper gives a general survey of multicast routing protocols in Mobile adhoc Networks (MANETs). The multicast routing protocols are divided into two categories- multicast routing based on application independence and multicast routing based on application dependence. Multicast routing protocols plays an important role in MANETs to provide group communication. Multicasting is one of the major communication technologies primarily designed for bandwidth conservation and an efficient way of transferring data to a group of receivers in wireless mesh networks.
\end{abstract}

\section{Keywords}

Mobile Ad-hoc Networks (MANETs), multicast routing protocols, tree based Protocols, Mesh-Based Protocols and Hybrid protocols.

\section{INTRODUCTION}

MANET is collection of independent mobile nodes that can communicate to each other via radio waves [1]. The communications between these mobile nodes are only possible if they are in radio range. In early days MANETs were called "packet radio" networks, which are sponsored by DARPA in 1970. The Internet predated by these packet radios and was part of motivation of the original IP suite. Applications of MANETs are -

There are some applications of MANETs-

- Search and rescue operations

- Military conflicts

- Immediate collaborative computing

- Urgent business meetings

- Personal area network and blue-tooth

Salient features of MANETS

- Dynamic Topologies

- Bandwidth Constrained links

- Energy Constrained Operation

- $\quad$ Limited Physical Security

\author{
Kushagra Agrawal \\ Department of Computer Science \& Engineering \\ Maharishi Markandeshwar University \\ Mullana, Ambala
}

\subsection{Multicasting}

The term multicasting means "Point-to-multipoint" or "one to many". In multicasting there is one source and multiple receivers, means copy of same data can be transmit to multiple receivers at a same time (see Figure.1). Due to this communication cost is reduced and efficiency of wireless channels is also improved. There are many benefits of multicasting in MANETs.

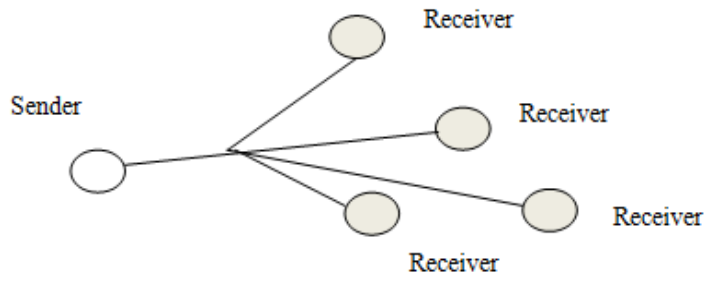

Fig 1: Multicast

Benefits of Multicast:

- Delivery to destinations simultaneously

- Deliver the messages over each link of the network only once

- only create copies when the links to the destinations split

\section{CLASSIFICATION OF MULTICAST ROUTING PROTOCOLS}

Application Independent- Most of the routing protocols is application Independent. In this hop count is use as a metric, means if the multiple paths are available to reach the destination then the minimum hop distance will be selected.

Application Dependent- Application Dependent multicast protocols are meant for only specific applications for which they are designed.

Based on Topology- Under this there are two categories-Tree Based and Mesh Based.

- Tree Based- In tree-based multicast routing protocols there is only one path between source and receiver. Tree based routing protocols are not robust to operate in highly mobile environment [2]. A treebased multicast routing protocol establishes and maintains a shared multicast routing tree to deliver data from a source to receivers of a multicast group [13].Tree Based multicast routing protocol is further divided into two types-Source Tree Based and Shared Tree Based. 
- $\quad$ Source Tree Based-In Source Tree Based each source node maintains a separate tree and all the receivers lies under this node [3].

- Shared Tree Based- In this a single tree is shared by all the sources in multicast group.

- Mesh Based- In mesh based multicast routing protocols there is more than one path between source and receiver pair. If any link failure occurs then these redundant paths are very useful, and provide higher packet delivery ratio [3].

Classifications of multicast routing protocols are shown in Figure 2.

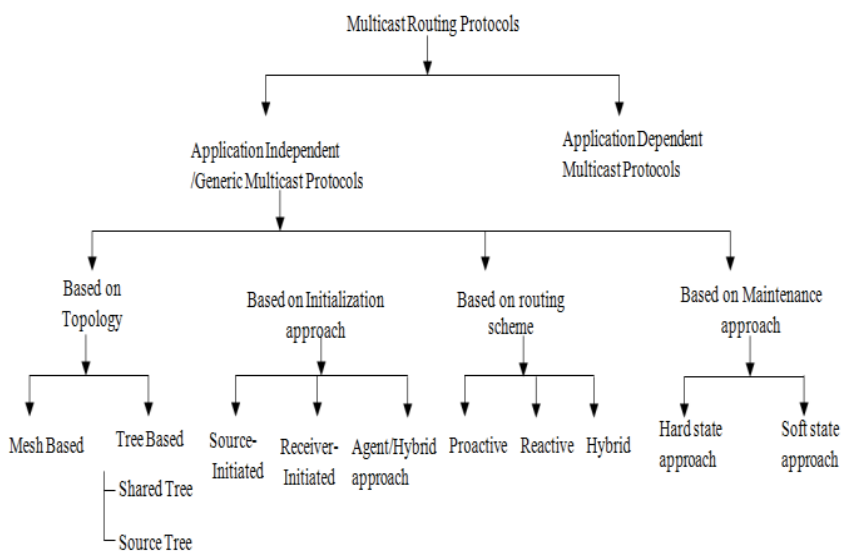

Fig 2: Classification of Multicast Routing Protocols

Based on Initialization approach- In this formation of multicast group can be initiated by source node as well as receiver node. Under this there are three approaches, namelySource Initiated, Receiver Initiated and Agent/Hybrid approach.

- Source Initiated- In Source Initiated the formation of multicast group is started by source node.

- Receiver Initiated- In Receiver Initiated the formation of multicast group is started by receivers of multicast group.

- Hybrid approach- In this construction and maintenance of multicast group done by either source node or receiver node. This approach basically combination of these two approaches i.e. Source initiated and receiver initiated.

Based on Routing scheme- Under this there are three approaches, namely-Reactive, Proactive and Hybrid approach.

- Reactive approach- Reactive approach also known as the on - demand approach. In this route is established on the basis of demand. When a node wants to communicate with other node and there is no route, then these routing protocols will try to establish the route.

- Proactive approach- Proactive approach also known as the table driven approach, in which each node maintain the network topology information in the form of routing tables. And these tables are periodically exchange to get the up to date view of the network.

- Hybrid approach- Hybrid approach is the combination of both these approaches. It is designed to overcome the limitation of these approaches (Proactive and Reactive approaches).

Based on Maintenance approach- There are two main approaches which is used for maintenance of multicast topology. This can be done by either soft state approach or either hard state approach.

- Soft- State approach- Soft- State(SS) approach is also known as "connectionless approach" .In this the control packets are flooded periodically to refresh the route, which cause a high packet delivery ratio at the cost of more control overhead.

- Hard- State approach- Hard- State (HS) approach is also known as "connection oriented approach". In this the control packets are only transmitted when a link breaks, due to this low control overhead but at the cost of low packet delivery ratio.

\subsection{Classification of Topology based Routing Protocols}

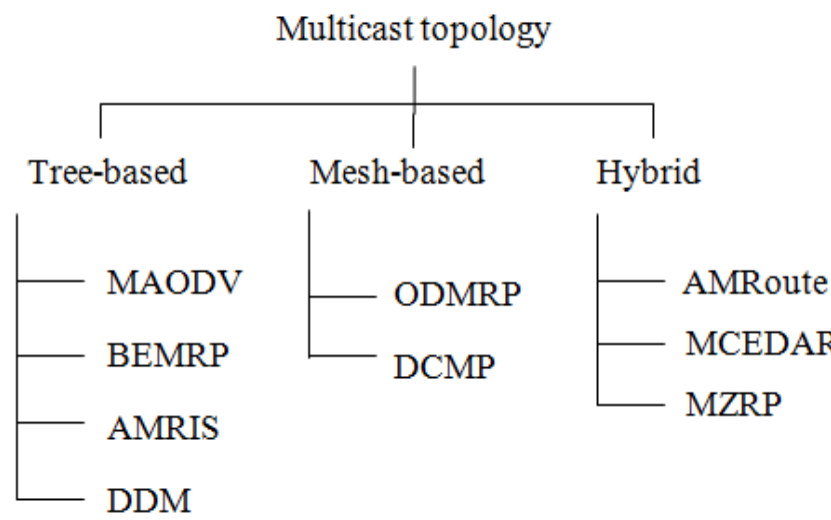

Fig 3: Classification of Topology based Routing Protocols

\subsubsection{Tree Based Multicast Routing Protocols-}

The following sections describe the tree based multicasting routing protocols. See Figure. 3

\subsubsection{Multicast Ad-hoc On-demand Distance Vector (MAODV)}

MAODV stands for Multicast Ad-hoc On-Demand Distance Vector Routing Protocol. This is the multicast extension of AODV.MAODV is a shared tree on-demand protocol. MAODV is proposed by Royer and Perkins in 1999. MAODV construct the shared tree more efficiently and it has low control overhead. MAODV support all the capabilities like unicast, multicast and broadcast. In MAODV each node maintains three tables-Routing Table (RT), Multicast Routing Table (MRT) and Request Table. The function of Request Table is same as that of in AODV, to store routing information. The maintenance of multicast group sequence no. is done by the group leader node. Figure 4 shows the working of MAODV.

Advantages- 1.The construction of multicast tree can be done quickly and efficiently by using unicast route information.

Disadvantages- 1 .Long delay 2.In highly mobility scenario low packet delivery ratio. 3. Not flexible, due to dependency on AODV 4.Single point failure problem. 


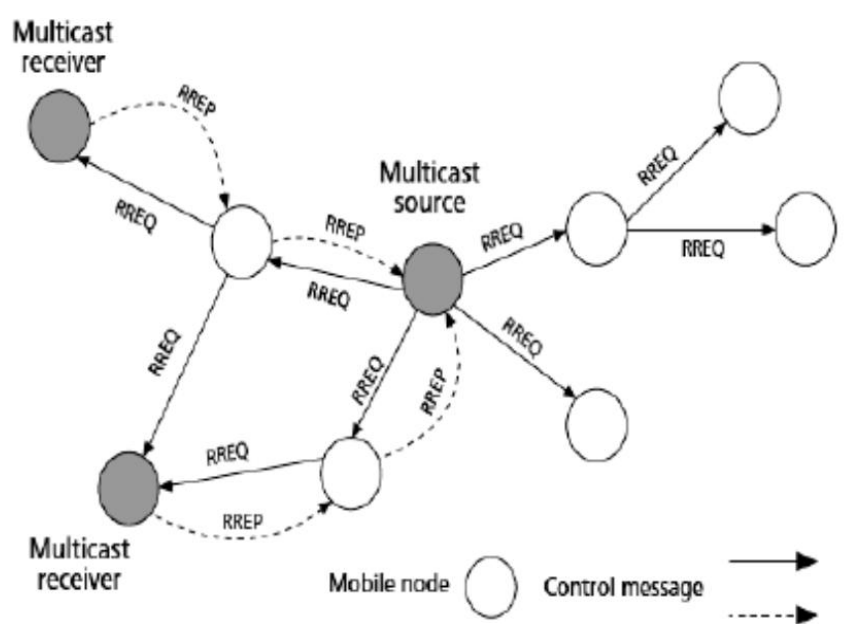

Fig 4: Discovery of Path in the MAODV Protocol

\subsubsection{Bandwidth Efficient Multicast Routing} Protocol (BEMRP)

Bandwidth Efficient Multicast Routing Protocol (BEMRP) is source-tree multicast routing protocol. There are three main phases-Tree-Initialization phase, Tree-Maintenance phase and Route Optimization phase. In Tree-Maintenance phase there are two schemes are used to rejoin the group. That areBroadcast-multicast scheme and Local rejoin scheme. In Broadcast-multicast scheme the upstream node floods broadcast-multicast packet and when isolated node receives this packet and rejoin the group. In Local rejoin scheme the isolated node floods Join control packet and upstream nodes send back reply packet when isolated node receives this reply and rejoin the group. See (Figure 5).

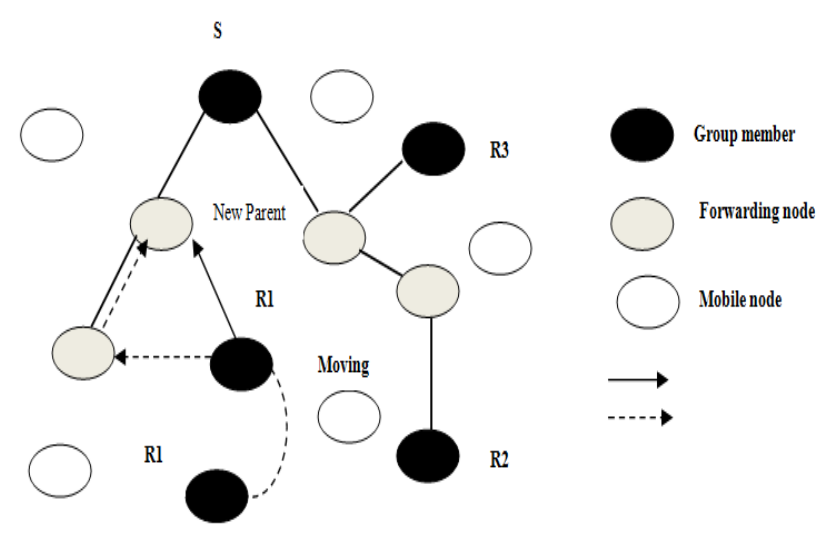

Fig 5: The process of route optimization in BEMRP

Advantages- 1.Bandwidth efficient

Disadvantages- 1.Long distance between source and receiver 2.High probability of path breaks 3.High delay

\subsubsection{Ad Hoc Multicast Routing Protocol \\ Utilizing Increasing Id-numbers (AMRIS)}

AMRIS is an On-Demand shared tree based multicast protocol. Multicast session member-id (msm-id) is assign to each node, which indicates the logical height of node in multicast delivery tree rooted at the sender that has the smallest msm-id (s-id) in the tree [1]. Sid is defined as the node that initiated the multicast session by broadcasting the
NEW-SESSION message to its surrounding nodes. In this there are two stages-Initialization and Maintenance. Figure 6 shows the working of AMRIS.

Advantages- 1.Simple topology 2.low overhead

Disadvantages- 1 .sensitive to mobility 2 .low delivery ratio 3.link breaks are not detected in time. 4. The usage of periodic beacons consumes bandwidth [9].

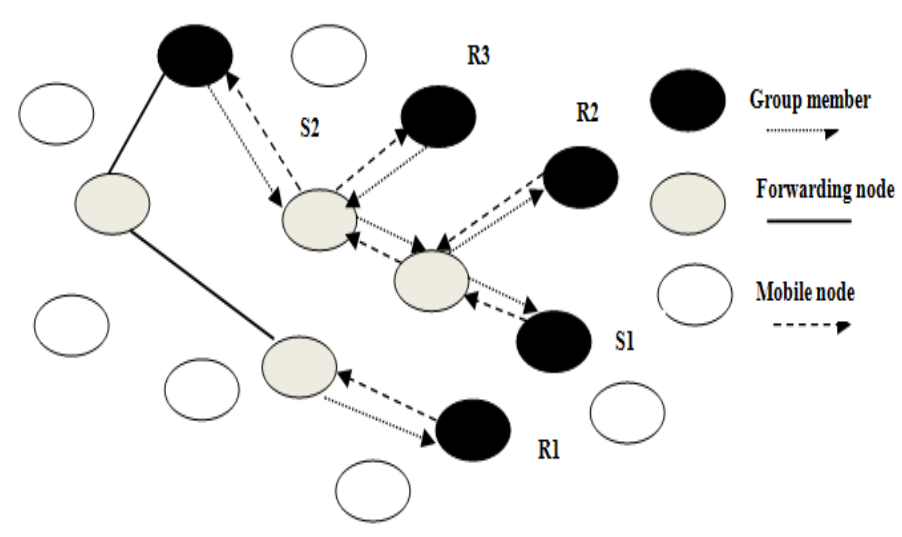

Fig 6: Procedure of joining in AMRIS

\subsubsection{The Differential Destination Multicast}

Protocol (DDM)

The Differential Destination Multicast (DDM) is an efficient source (sender) based tree approach, designed for only small multicast group. DDM employs two types of packets: control packets and data packets, where it is understood that data packets may also contain control information. In this protocol, source node has the knowledge of all the members of the multicast group as their information is embedded in the data packets which are to be transferred to the destinations [1]. DDM has two modes-Stateless and Soft State. It has also Explicit Header means all destinations are placed in the packet headers. There are four types of control packets: JOIN, ACK, LEAVE and RSYNC [7]. See (Figure 7).

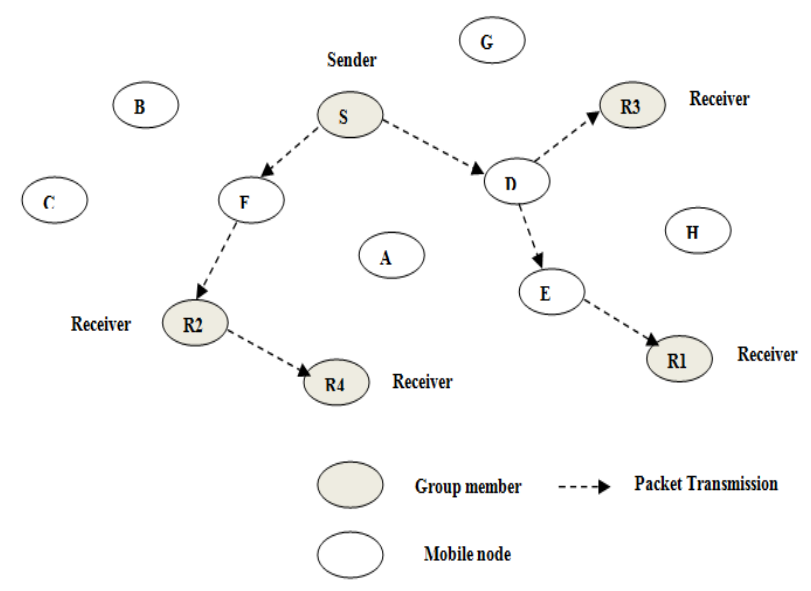

Fig 7: The transmission Packet in DDM

\subsubsection{Mesh-Based Multicast Routing Protocols-}

The following section describes about the Mesh based multicasting protocols with its advantages and disadvantages. 


\subsubsection{On-Demand Multicast Routing Protocol (ODMRP)}

ODMRP- On-Demand Multicast Routing Protocol (ODMRP) is proposed by Bae at el in 2001. ODMRP maintain mesh topology rooted from each source. ODMRP has more than one path between sender and receiver rather than MAODV which is tree based. The routes from source to receivers build a mesh of nodes which is called "Forwarding Group". ODMRP broadcast the reply back to the source. The difference between ODMRP and MAODVA is MAODV does not activate a multicast route immediately while ODMRP does. In ODMRP there are two multicast messages-JOINQUERY and JOIN-REPLY. And it has two phases-Request phase and Reply phase. See (Figure 8).

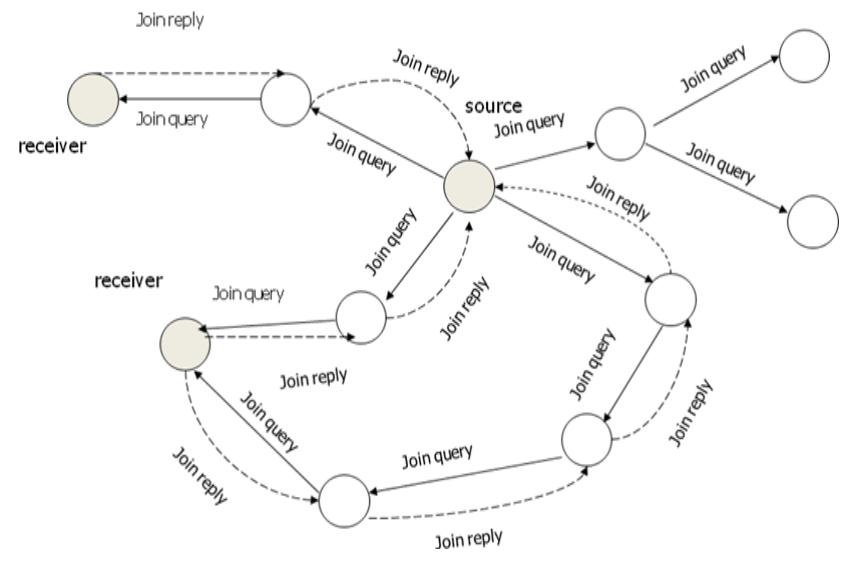

Fig 8: Mesh creation in ODMRP

Advantages - 1. Simplicity 2.Robustness to host mobility 3.Higher packet delivery ratio 3.Unicast routing capability

Disadvantages- 1.Complex topology 2.High overheads

\subsubsection{Dynamic Core Based Multicast Routing Protocols (DCMP)}

In DCMP there are different categories of sources. These sources are active sources, core active sources and passive sources. The Active sources are same as the sources of ODMRP in which they flood Join-Request control packets at regular interval of time. Core Active sources are those Active sources which act as core for one or more Passive sources. A Passive source depends on a nearby Active source for forwarding its data packets [10]. See (Figure 9).

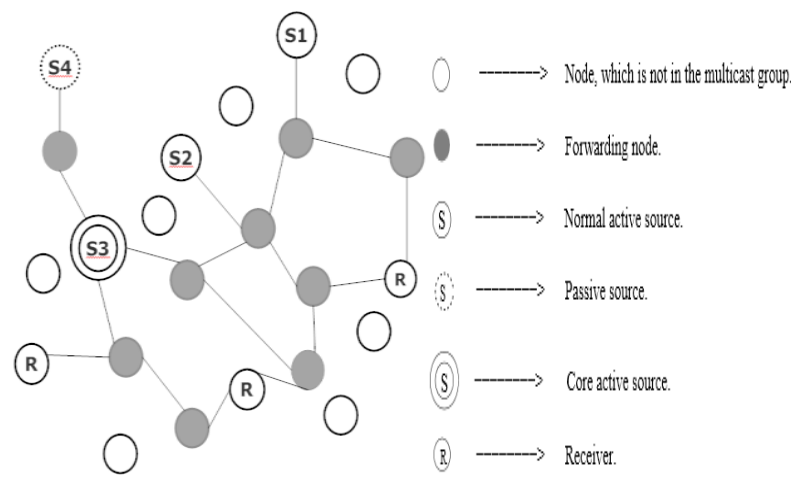

Fig 9: Construction of mesh in DCMP

Advantages - 1.Scalability due to decreased control overhead 2.High packet delivery ratio
Disadvantages 1.Path stability is decreased. 2. If core node failure, due to this many passive sender suffer.

\subsubsection{Hybrid Multicasting}

In Hybrid approach it is the combination of Mesh and Treebased approaches. This attains both the robustness and efficiency.

\subsubsection{Ad-Hoc Multicast Routing Protocol (AMRoute)}

AMRoute is bidirectional shared-tree means one tree per group and creates a mesh to establish connectivity before tree creation. There are two phases-mesh creation and treecreation [6].Only group sender and receiver are tree nodes (replication/forwarding only performed by group members and state only in tree node).No support needed from network nodes who are not interested/capable of multicast. AMRoute uses five control messages- JOIN_REQ, JOIN_ACK, JOIN_NAK, TREE_CREATE, TREE_CREATE_NAK and DATA_MESSAGE [8]. See (Figure 10).

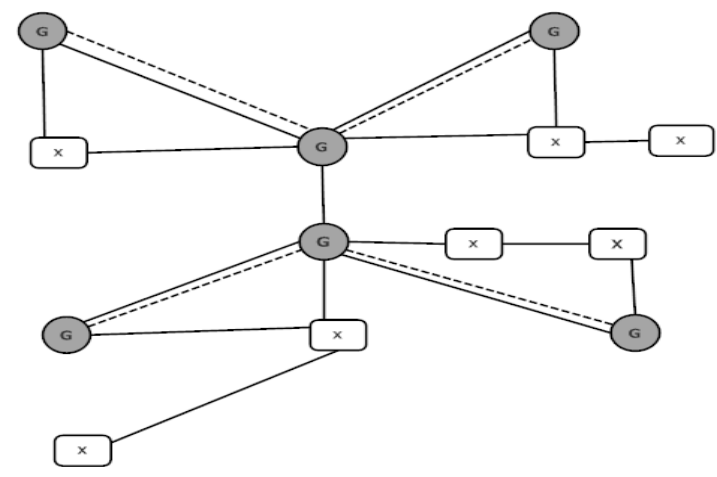

Fig 10: Ad hoc Multicast Routing Protocol

Advantages-1.Uses virtual mesh links to establish the multicast tree [11].

Disadvantages- 1.loops exist and non-optimal trees are built 2.suffer from single point of failure of the core node [7]. 3.High overhead 4.Uses virtual mesh links to establish the multicast tree.

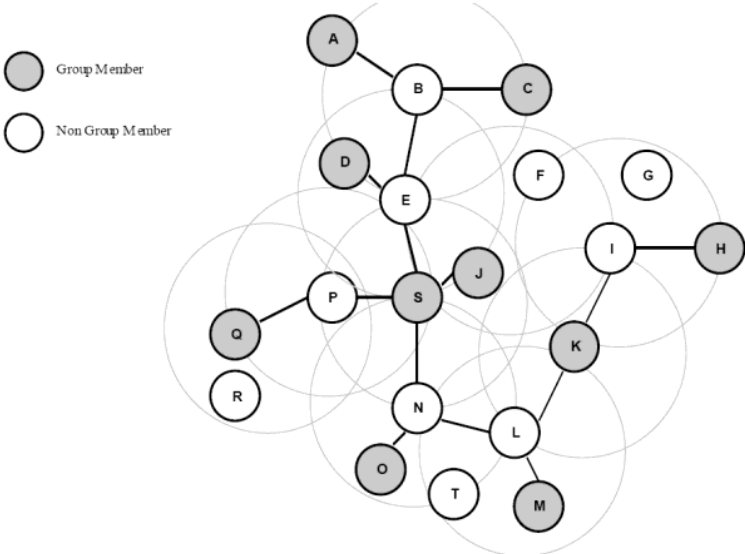

Fig 11: Extension of the Multicast Tree to the Entire Network (adapted from [6]) 


\subsubsection{Multicast Core-Extraction Distributed Ad Hoc Routing (MCEDAR)}

The multicast extension of the unicast Zone Routing Protocol (ZRP) is MZRP. It is combination of both strategies like proactive and Reactive routing strategies. Proactive routing uses excess bandwidth to maintain routing information, while reactive routing involves long route request delays [5].In this there are two phases-Tree-Initialization phase and TreeMaintenance phase. In Tree-Initialization phase the multicast tree is created inside the zone by sending Tree-Create message and receiving node send Tree-Create-Ack message. To extend the multicast tree the Tree-Propagate message is used. In Tree-Maintenance phase the source node sends the Tree-Refresh periodically, if tree node does not receive this packet for a special period, then it is isolated. See (Figure 11).

Advantages-1.Reduce control overhead because it runs over Zone routing protocol

Disadvantages-1.Long waits for the far node because of the Tree-Propagate message

\subsubsection{Multicast Core-Extraction Distributed Ad Hoc Routing (MCEDAR)}

The multicast extension of CEDAR is MCEDAR that provides the robustness of mesh structures and the efficiency of tree structures. MCEDAR uses a mesh as the underlying infrastructure, in this the data forwarding occurs only on a sender-rooted tree [6]. There are two aspects- Route Management and Data forwarding. It does not differentiate between the senders and receivers of a multicast group [1]. In this node who wants to join a group sends a JoinReq packet and node join the group by receiving JoinAck packet. While isolated, node issues a JoinReq again. MCEDAR use a new mesh structure for multicast routing that called as mgraph. See (Figure 12).

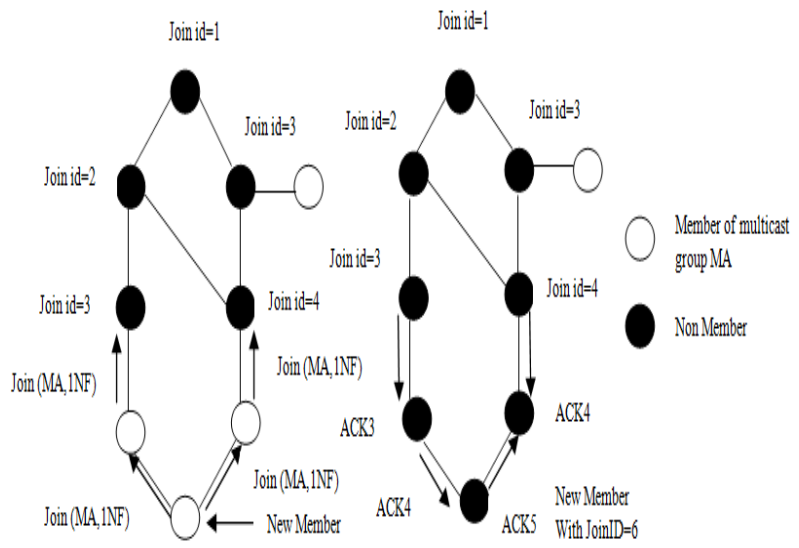

Fig 12: The Join Protocol

Advantages - 1.Robust 2.It is efficient as other Tree-Based protocols 3.Independent of the underlying unicast routing protocol.

Disadvantages - 1. Complex 2. Increasing control overhead

\section{SUMMARY}

Some of important multicast routing protocols which are designed for MANETs are explained in this paper. These routing protocols are classified into three categories: treebased protocols, mesh based protocols and hybrid based. Tree-based protocols are efficient in data transmission while mesh-based protocols are robust to topology changes and are more stable than tree-based protocols. All these protocols have their own advantages and disadvantages. Hybrid multicast which is tree based as well as mesh based and gives the advantage of both these types.

Table 1: Summary of multicast routing protocols

\begin{tabular}{|c|c|c|c|c|c|c|c|}
\hline $\begin{array}{c}\text { Protocol } \\
\text { Name }\end{array}$ & $\begin{array}{l}\text { Multicast } \\
\text { Topology }\end{array}$ & Initialization & $\begin{array}{l}\text { Independent of } \\
\text { Routing Protocol }\end{array}$ & $\begin{array}{c}\text { Dependency on } \\
\text { Specific Routing } \\
\text { Protocol }\end{array}$ & $\begin{array}{c}\text { Maintenance } \\
\text { Approach }\end{array}$ & $\begin{array}{c}\text { Periodic Control } \\
\text { Messaging }\end{array}$ & Loop Free \\
\hline MAODV & Shared-tree & Receiver & Yes & No & Hard state & Yes & Yes \\
\hline BEMRP & Source-tree & Receiver & Yes & $\mathrm{N}_{0}$ & Hard state & $\mathrm{N}_{0}$ & Yes \\
\hline AMRIS & Shared-tree & Source & Yes & $\mathrm{N}_{0}$ & Hard state & Yes & Yes \\
\hline DDM & Source-tree & Receiver & $\mathrm{N}_{0}$ & $\mathrm{~N}_{0}$ & Sott state & Yes & Yes \\
\hline ODMRP & Mesh & Source & Yes & No & Sot state & Yes & Yes \\
\hline DCMP & Mesh & Source & Yes & No & Soft state & Yes & Yes \\
\hline AMRoute & $\begin{array}{l}\text { Source-tree over } \\
\text { Mesh }\end{array}$ & $\begin{array}{l}\text { Source or } \\
\text { Receiver }\end{array}$ & $\mathrm{N}_{0}$ & $\mathrm{~N}_{0}$ & Hard state & Yes & Yes \\
\hline MCEDAR & $\begin{array}{c}\text { Source-tree over } \\
\text { Mesh }\end{array}$ & $\begin{array}{l}\text { Source or } \\
\text { Receiver }\end{array}$ & $\mathrm{N}_{0}$ & Yes (CEDAR) & Hard state & $\mathrm{N}_{0}$ & Yes \\
\hline MZRP & Source-tree & Source & Yes & $\mathrm{N}_{0}$ & Hard state & Yes & Yes \\
\hline
\end{tabular}

\section{REFERENCES}

[1] Parul Vashist and K.Hema, "New Multicast Routing Protocol In Ad-Hoc Network" in International Journal of Innovations in Engineering and Technology(IJIET),Vol.2 Issue2 April 2013.

[2] N.Fareena, A Shunmuga, Priya Mala and Dr.K.Ramar, "Mobility based energy efficient multicast protocol for MANET", in ICMOC 2012

[3] Ahmed. M. Abdel Mo'men, Haitham. S. Hamza, IEEE Member, and Iman. A. Saroit, "A Survey on Security Enhanced Multicast Routing Protocols in Mobile Ad Hoc Networks", in IEEE 2010

[4] Natarajan Meghanathan, "Survey of Topology-based Multicast Routing Protocols for Mobile Ad hoc Networks", in International Journal of Communication Networks and Information Security (IJCNIS), Vol. 3, No. 2, August 2011

[5] V.Mallaiah,Dr.A.VinayaBabu,K.Madhukar,S.Nagaprasd, D.Marlene Grace Verghese and A.Sreelatha "MULTICAST ZONE ROUTING PROTOCOL IN WIRELESS MOBILE ADHOC NETWORKS", in International Journal of Engineering Science and Technology, Vol. 2(10), 2010

[6] CHEN-CHE HUANG, SHOU-CHIH LO and TAIWAN, "A Comprehensive Survey of Multicast Routing Protocols for Mobile Ad Hoc Networks".

[7] Lusheng Ji and M.Scott Corson, "Differential Destination Multicast-A MANET Multicast Routing Protocol for Small Groups, in IEEE INFOCOM 2001.

[8] JASON XIE, MINGYAN LIU, RAJESH R. TALPADE and ANTHONY MCAULEY, "AMRoute: Ad Hoc Multicast Routing Protocol",in Mobile Networks and Applications 7, 429-439, 2002 
[9] Velumani R and Dr. K. Duraisamy, "A Concise Survey on Multicast Routing Protocols for MANETs", in International Journal of Advanced Research in Computer Science and Software Engineering, Volume 2, Issue 5, May 2012

[10] Subir Kumar Das, B. S. Manoj and C. Siva Ram Murthy, "A Dynamic Core Based Multicast Routing Protocol for Ad hoc Wireless Networks", MOBIHOC'02,June 9-11, 2002, EPFL Lausanne.

[11] B. Ravi Prasad, Dr.A.Damodaram and Dr.G.Venkateswara Rao, "Performance Comparison of Different Multicast Routing Protocols in Mobile Ad-hoc Networks", in International Journal of Engineering
Research \& Technology (IJERT), Vol. 1 Issue 5, July 2012.

[12] Aarti and Dr. S. S. Tyagi, "Study of MANET: Characteristics, Challenges, Application and Security Attacks", in International Journal of Advanced Research in Computer Science and Software Engineering, Volume 3, Issue 5, May 2013.

[13] Rajneesh Gujral, Sanjeev Rana and Amrita Chaudhary, "Study and Comparison of Mesh and Tree-Based Multicast Routing Protocols for MANETs", in International Journal of Latest Trends in Engineering and Technology (IJLTET), Vol. 1, Issue 2, July 2012 